\title{
SFMBT2 positively regulates SOX9 and chondrocyte proliferation
}

\author{
SAFDAR HUSSAIN ${ }^{1,2}$, MENGYAO SUN $^{1}$, YUANXU GUO $^{1}$, NOSHEEN MUSHTAQ $^{3}$, YITONG ZHAO $^{1}$, \\ YING YUAN $^{1}$, NAZIM HUSSAIN ${ }^{2}$, EZRA OSORO ${ }^{1}$, ABUBAKAR SULEIMAN $^{1}$, MUHAMMAD SADIQ ${ }^{1}$,

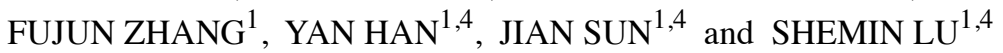 \\ ${ }^{1}$ Department of Biochemistry and Molecular Biology, School of Basic Medical Sciences, Xi'an Jiaotong University, \\ Health Science Center, Xi'an, Shaanxi 710061, P.R. China; ${ }^{2}$ Centre for Applied Molecular Biology (CAMB), \\ University of The Punjab, Lahore 53700, Pakistan; ${ }^{3}$ Department of Microbiology, School of Basic Medical Sciences, \\ Xi'an Jiaotong University, Health Science Center; ${ }^{4}$ Key Laboratory of Environment and Genes Related to Diseases, \\ Ministry of Education, Xi'an, Shaanxi 710061, P.R. China
}

Received June 18, 2018; Accepted September 19, 2018

DOI: $10.3892 /$ ijmm.2018.3894

\begin{abstract}
SRY-box 9 (SOX9) is the master regulator of the chondrocyte phenotype, which is essential for differentiating chondrogenic mesenchymal condensations into chondrocytes, and is involved in regulating every stage of chondrocyte differentiation. SOX9 deletion in chondrocytes at the late stages of cartilage development results in decreased chondrocyte proliferation; inhibited expression of cartilage matrix genes, including Indian hedgehog and the downstream parathyroid hormone-related protein; and premature conversion of proliferating chondrocytes into hypertrophic chondrocytes, which mineralize their matrix prematurely. Therefore, SOX9 is considered vital for the majority of phases of chondrocyte lineage, from early condensations to the differentiation of proliferating chondrocytes, leading to chondrocyte hypertrophy. It has been reported that SOX9 expression is decreased in osteoarthritis (OA) cartilage. Regeneration or repair of cartilage degradation in OA remains a challenge. Previous studies have indicated that overexpression of SOX9 can promote cartilage repair and can be used as a potential therapeutic agent at the early stages of human OA. The present study identified Scm-like with four malignant brain tumor domains 2 (SFMBT2) as a novel regulator of SOX9 expression in human chondrocytes. Our previous study revealed that SFMBT2 is negatively regulated in OA cartilage, and decreased levels of SFMBT2 contribute to the catabolic phenotype of chondrocytes. The present study detected increased
\end{abstract}

Correspondence to: Dr Jian Sun or Dr Shemin Lu, Department of Biochemistry and Molecular Biology, School of Basic Medical Sciences, Xi'an Jiaotong University, Health Science Center, 76 Yanta West Road, Xi'an, Shaanxi 710061, P.R. China

E-mail: sunjian1@mail.xjtu.edu.cn

E-mail: lushemin@mail.xjtu.edu.cn

Key words: SFMBT2, SOX9, chondrocyte, proliferation, osteoarthritis expression levels of SFMBT2 in early cartilage development and during the early phases of chondrogenesis. Overexpression of SFMBT2 in C28/I2 cells upregulated SOX9 expression in a dose-dependent manner. Furthermore, SFMBT2 positively regulated C28/I2 cell proliferation and restored the decreased levels of SOX9 in chondrocytes following tumor necrosis factor- $\alpha$ treatment. Additional studies may reveal novel insights into the molecular mechanism involved and the potential role of SFMBT2 in cartilage repair and OA management.

\section{Introduction}

Mature chondrocytes are metabolically slow, highly differentiated cells with a limited proliferation rate and, unlike other tissues such as bone, a restricted ability to self-repair cartilage degeneration $(1,2)$. Despite their low numbers and being the sole cell type of the cartilage, chondrocytes serve fundamental roles in regulating tissue properties, such as organization, remodeling, synthesis and degradation of the surrounding matrix $(3,4)$. During skeletal development, chondrocytes arise from mesenchymal progenitors to synthesize the templates or cartilage anlagen for the developing bone (5). Chondrogenesis occurs as a result of condensation of mesenchymal cells. The process can be generally divided into two phases: i) Early chondrogenesis, which is characterized by upregulated collagen type II $\alpha 1$ chain (COL2A1) and SRY-box 9 (SOX9), and ii) hypertrophy, which is characterized by upregulated collagen type $\mathrm{X} \alpha 1$ chain (COL10A1) and runt-related transcription factor 2 . These two stages are regulated by numerous factors and signaling pathways (6), and they function to repress each other (7-10).

The metabolic behavior of chondrocytes can be modified by various factors, including aging, genetic makeup, and other mechanical and chemical factors, such as joint loading and inflammation. Proinflammatory cytokines, including tumor necrosis factor- $\alpha$ (TNF- $\alpha$ ) and interleukin-1 $\beta$ (IL-1 $\beta$ ) exert catabolic and anabolic effects, and serve important roles in the degradation and synthesis of the extracellular matrix (ECM) (11). In osteoarthritis (OA), cartilage deterioration is associated with disruption of the metabolic homeostasis of 
chondrocytes and dysregulation of their physiological behavior, which is reflected by the appearance of cartilage surface fibrillation, formation of chondrocyte clusters and alterations in the composition of ECM components (12).

SOX9 is considered the master regulator and a key transcriptional factor in chondrocyte condensation, proliferation and differentiation (13-16). In chondrogenesis, SOX9 acts as a homo-dimer (17), which is stabilized by two other SOX members of the SoxD family: L-SOX5 and SOX6 (18). These molecules are co-expressed with SOX9 during cell differentiation, and are associated with the production of collagen types II and IX, as well as aggrecan (ACAN), which is the major proteoglycan (19). SOX9 is expressed in all prechondrocytic and chondrocytic cells during mouse embryonic development, whereas its expression is completely abolished in hypertrophic chondrocytes (20-22). Mutations in the SOX9 gene lead to campomelic dysplasia, a disease characterized by skeletal malformation and 46,XY sex reversal (23).

Polycomb group ( $\mathrm{PcG}$ ) proteins are known to regulate developmental genes, and abnormal expression of these proteins may cause cancer $(24,25)$. Scm-like with four malignant brain tumor (MBT) domains 2 (SFMBT2) is a member of the PcG protein family (26), which has been reported to be involved in prostate cancer cell growth, through repression of the homeobox B13 gene in DU145 cells (27). SFMBT2 consists of a sterile $\alpha$ motif domain and four MBT domains, which are crucial for gene regulation by recognizing and binding to methylated lysine residues in the tails of histones $\mathrm{H} 3$ and H4 (28). However, to the best of our knowledge, no study regarding its role in cartilage or OA has been published to date.

Our previous study screened differentially expressed genes in OA cartilage using the suppression subtractive hybridization technique, and identified SFMBT2 as a candidate gene from the reverse-subtracted cDNA library by sequence analysis and similarity search with BLAST (29). Our recent study verified the negative association of this gene with OA by analyzing the expression of SFMBT2 in damaged cartilages from human patients with OA compared with normal cartilages from control individuals, and established that the down-regulation of SFMBT2 contributes to the catabolic phenotype of chondrocytes (30). The aim of the present study was to examine the expression of SFMBT2 in cartilage at different stages of its development, and during the process of chondrogenesis. Understanding the mechanisms underlying cartilage remodeling during development, $\mathrm{OA}$ and aging, and identifying the novel factors involved in these mechanisms may lead to the development of more effective strategies, preventing cartilage damage and promoting repair.

\section{Materials and methods}

Rats. Dark agouti (DA) rats, which originated from Medical Inflammation Research, Lund University (Lund, Sweden), were bred and maintained in polystyrene cages containing sterile wood shavings in a specific pathogen-free animal house at the Department of Biochemistry and Molecular Biology, Xi'an Jiaotong University (Xi'an, China). Rats were fed standard rodent chow and water ad libitum and were maintained in a climate-controlled environment (temperature:
20-26 ${ }^{\circ} \mathrm{C}$; relative humidity: $40-70 \%$ ) under a $12 \mathrm{~h}$ light/dark cycle. Femoral head cartilages were surgically removed from 12 age/weight-matched rats, four for each developmental stage (two females, two males), after sacrificing the rats on postnatal days 0 (D0), 21 (D21) and 42 (D42). The cartilage samples were immediately placed in liquid nitrogen after cleaning with PBS and were stored at $-80^{\circ} \mathrm{C}$ for further analysis. The experiments were approved by the Institutional Animal Ethics Committee of Xi'an Jiaotong University (Xi'an, China).

Cell culture. The ATDC5 murine chondroblast cell line was obtained from the European Collection of Authenticated Cell Cultures (Salisbury, UK) and was cultured in Dulbecco's modified Eagle's medium (DMEM)/Ham's F12 (HyClone; GE Healthcare Life Sciences, Logan, UT, USA) supplemented with 5\% fetal bovine serum (FBS; Shanghai ExCell Biology, Inc., Shanghai, China). Chondrogenesis was induced by adding ITS-supplement (insulin, $1 \mathrm{mg} / \mathrm{ml}$; transferrin, $0.55 \mathrm{mg} / \mathrm{ml}$; and selenium, $0.5 \mu \mathrm{g} / \mathrm{ml}$ ) directly into the medium. The medium was changed every 2 nd or 3 rd day. The cells were harvested for RNA and protein isolation on D0, D3, D7 and D14 post-induction.

The C28/I2 human juvenile costal chondrocyte cell line was kindly provided by Prof. Junling Cao (Institute of Endemic Diseases, Xi'an Jiaotong University, Health Science Center, Xi'an, China). Chondrocytes were cultured in DMEM/F12 containing 10\% FBS and 1\% penicillin-streptomycin (Sigma-Aldrich; Merck KGaA, Darmstadt, Germany). Both chondrocyte lines were maintained at $37^{\circ} \mathrm{C}$ in the presence of $5 \% \mathrm{CO}_{2}$. Once the cells reached $90 \%$ confluence, they were detached by treatment with $0.05 \%$ trypsin (Shanghai ExCell Biology, Inc.) and passaged.

Transient transfection/infection with small interfering RNAs (siRNAs)/adenovirus vectors. For the knockdown experiments, siRNAs against SFMBT2 (si-SFMBT2) and SOX9 (si-SOX9), and the scrambled negative control siRNA (si-NC), were synthesized (Shanghai GenePharma Co., Ltd., Shanghai, China). C28/I 2 cells were seeded in 12 -well plates $\left(1 \times 10^{5}\right.$ cells/well), after which, they were transfected with the $80 \mathrm{nM}$ si-SFMBT2, $60 \mathrm{nM}$ si-SOX9 or $60 \mathrm{nM}$ si-NC using Lipofectamine ${ }^{\circledR} 2000$ (cat. no. 11668-019; Invitrogen; Thermo Fisher Scientific, Inc., Waltham, MA, USA), according to the manufacturer's protocol. Briefly, C28/I2 cells were incubated with transfection complexes in serum-free medium for $4 \mathrm{~h}$ at $37^{\circ} \mathrm{C}$ in a $\mathrm{CO}_{2}$ incubator. After $4 \mathrm{~h}$, the medium was replaced with fresh, complete medium (containing $10 \% \mathrm{FBS}$ ), and cells were incubated at $37^{\circ} \mathrm{C}$ in the presence of $5 \% \mathrm{CO}_{2}$. Effects of siRNA interference were determined by reverse transcription-quantitative polymerase chain reaction (RT-qPCR) and western blotting 12-72 h post-transfection, as indicated. The siRNA sequences used in the present study were as follows: si-NC, sense, UUCUCCGAACGUGUC ACGUTT and antisense, CGUGACACGUUCGGAGAATT; si-SFMBT2, sense, 5'-GCACUUUGUCAGCUUCCAATT-3' and antisense, 5'-UUGGAAGCUGACAAAGUGCTT-3'; and si-SOX9, sense, 5'-GCAGCGACGUCAUCUCCAAdTdT-3' and antisense, 5'-CGUCGCUGCAGUAGAGGUUdTdT-3'.

The FLAG-green fluorescent protein-tagged SFMBT2 adenovirus vector (ad-SFMBT2; $\left.1 \times 10^{10} \mathrm{PFU} / \mathrm{ml}\right)$ and the control adenovirus vector (ad-NC; $\left.2 \times 10^{10} \mathrm{PFU} / \mathrm{ml}\right)$ were 
purchased from Hanbio Biotechnology Co., Ltd. (Shanghai, China). For overexpression of SFMBT2, C28/I2 cells were seeded in 12-well plates $\left(1 \times 10^{5}\right.$ cells/well) or 96 -well plates $\left(1 \times 10^{4}\right.$ cells/well), and were incubated with ad-NC or ad-SFMBT2, which were added directly into the medium at the indicated titers/multiplicities of infection (MOIs) for $4 \mathrm{~h}$ at $37^{\circ} \mathrm{C}$ in a $\mathrm{CO}_{2}$ incubator. After $4 \mathrm{~h}$, the medium containing the viral vectors was removed and replaced with fresh medium, and the cells were incubated at $37^{\circ} \mathrm{C}$ in the presence of $5 \% \mathrm{CO}_{2}$. The chondrocytes were harvested at the indicated time intervals (0-72 h) after adenoviral infection for RNA and protein extraction.

Chondrocyte treatment with TNF- $\alpha$ and IL-1 $\beta$. C28/I2 cells were serum starved for $10 \mathrm{~h}$ prior to the addition of TNF- $\alpha$ $(10 \mathrm{ng} / \mathrm{ml})$ or IL-1 $\beta$ (10 ng/ml) (both from Sino Biological Inc., Beijing, China) into the culture medium. The cells were then incubated at $37^{\circ} \mathrm{C}$ in the presence of $5 \% \mathrm{CO}_{2}$ and were harvested for protein isolation $24 \mathrm{~h}$ post-treatment. C28/I2 cells infected with ad-NC or ad-SFMBT2 were treated with TNF- $\alpha 24$ h post-adenoviral infection, and were harvested for protein isolation at the indicated time points $(0-48 \mathrm{~h})$ following TNF- $\alpha$ treatment.

Total RNA extraction and RT- $q P C R$. Total RNA was extracted from the cultured chondrocytes $\left(1 \times 10^{5}\right.$ cells/well) using TRIzol ${ }^{\circledR}$ reagent (Invitrogen; Thermo Fisher Scientific, Inc.) and was reverse transcribed to cDNA using the RevertAid First Strand cDNA Synthesis kit (Thermo Fisher Scientific, Inc.), according to the manufacturer's protocol. Gene expression levels were detected by qPCR (Agilent Stratagene Mx3005; Agilent Technologies, Inc., Santa Clara, CA, USA) using the SYBR Green system (Roche Diagnostics, Indianapolis, IN, USA). Thermocycling conditions were as follows: $95^{\circ} \mathrm{C}$ for 10 min (activation), followed by 40 cycles at $95^{\circ} \mathrm{C}$ for $15 \mathrm{sec}$ (denaturation), $61 / 64^{\circ} \mathrm{C}$ for $30 \mathrm{sec}$ (annealing) and $72^{\circ} \mathrm{C}$ for $30 \mathrm{sec}$ (extension). Data collection was performed during each extension phase. Relative quantification of the target genes, normalized to the control, was calculated using the comparative $\mathrm{Cq}(\Delta \Delta \mathrm{Cq})$ method (31). The primers used for RT-qPCR analysis are listed in Table I.

Protein isolation and quantification. Total protein was isolated from the cultured chondrocytes $\left(1 \times 10^{5}\right.$ cells/well) using radioimmunoprecipitation assay lysis buffer (Beyotime Institute of Biotechnology, Haimen, China) containing protease and phosphatase inhibitor mixture (Bimake, Houston, TX, USA). The whole cell lysates were incubated on ice for $30 \mathrm{~min}$, centrifuged at $12,000 \mathrm{x}$ g for $15 \mathrm{~min}$ at $4^{\circ} \mathrm{C}$ to remove cellular debris, and the supernatants were transferred to clean $1.5 \mathrm{ml}$ tubes. Protein concentration in the cleared lysates was determined by Bicinchoninic Acid Protein Assay (Tiangen Biotech Co., Ltd., Beijing, China).

Protein sample preparation and immunoblotting. The clear lysates were mixed with SDS loading dye and heated at $95^{\circ} \mathrm{C}$ for $5 \mathrm{~min}$. Subsequently, $20 \mu \mathrm{g}$ proteins were separated by $10 \%$ SDS-PAGE and transferred to the polyvinylidene fluoride membrane (EMD Millipore, Billerica, MA, USA). The membrane was blocked in $5 \%$ non-fat milk or $5 \%$ bovine serum albumin (BSA, Amresco; VWR, Radnor, PA, USA) at room temperature for $2 \mathrm{~h}$, and then incubated at $4^{\circ} \mathrm{C}$ overnight with primary antibodies. After washing three times with Tris-buffered saline containing $0.1 \%$ Tween-20, the membrane was incubated with a horseradish peroxidase-conjugated secondary antibody for $2 \mathrm{~h}$ at room temperature. The blots were detected using the enhanced chemiluminescence detection system (EMD Millipore). Western blots were scanned using the GeneGnome XRQ system (Syngene, Frederick, MD, USA), and densitometry of the bands was analyzed with GeneTools analysis software V 4.01 (Syngene). The antibodies and their dilutions are shown in Table II.

Immunohistochemistry. Immunohistochemical staining was performed using the standard streptavidin peroxidase complex method. Cartilage tissues were fixed in $4 \%$ buffered paraformaldehyde for $>2$ days at room temperature, and were decalcified with buffered EDTA (12.5\% EDTA, $\mathrm{pH}$ 7.4) for 4 weeks at room temperature. Subsequently, the samples were dehydrated through a graded series of alcohol, embedded in paraffin and $5-\mu \mathrm{m}$ sections were obtained; the tissue sections were deparaffinized in xylene and rehydrated through a graded series of alcohol. Following treatment with $3 \% \mathrm{H}_{2} \mathrm{O}_{2}$ for $10 \mathrm{~min}$ at room temperature and enzymatic digestion (Antigen Retrieval Buffer, cat. no. AR0022; Wuhan Boster Biological Technology, Ltd.) for $10 \mathrm{~min}$ at $37^{\circ} \mathrm{C}$, tissue sections were blocked with $5 \% \mathrm{BSA}$ for $20 \mathrm{~min}$ at $4^{\circ} \mathrm{C}$ and were incubated with the anti-SFMBT2 primary antibody (Table II) overnight at $4^{\circ} \mathrm{C}$. Subsequently, sections were incubated with the biotinylated secondary antibody $(1: 1,000)$ for $30 \mathrm{~min}$ at $37^{\circ} \mathrm{C}$ and horseradish peroxidase-conjugated streptavidin $(1: 1,000)$ for $30 \mathrm{~min}$ at $37^{\circ} \mathrm{C}$, both of which were contained within the DAB substrate kit (Wuhan Boster Biological Technology, Ltd.). Sections were counterstained with hematoxylin and mounted with neutral balsam. Images of the staining were captured under a fluorescence microscope (Olympus BX51; Olympus Corporation, Tokyo, Japan) and staining was semi-quantified using Image-Pro ${ }^{\circledR}$ Plus (V6.0) software (Media Cybernetics, Inc., Rockville, MD, USA).

Cell proliferation assay. Cell Counting kit-8 (CCK-8; Bimake) assay was used to evaluate the proliferative abilities of chondrocytes. As previously reported (32), C28/I2 cells were seeded in 96-well plates ( $1 \times 10^{4}$ cells/well) in DMEM/Ham's F12 and were maintained at $37^{\circ} \mathrm{C}$ in the presence of $5 \% \mathrm{CO}_{2}$. Subsequently, cells were transfected/infected with si-SFMBT2/ad-SFMBT2, alongside the respective controls (si-NC/ad-NC). At 12, 24, 48 and $72 \mathrm{~h}$ post-transfection/infection, $10 \mu \mathrm{l} \mathrm{CCK-8} \mathrm{solution} \mathrm{was}$ added directly into each well and the cells were incubated for a further $4 \mathrm{~h}$. Absorbance was measured at $450 \mathrm{~nm}$ using a microplate reader (Thermo Scientific Multiskan ${ }^{\circledR}$ Spectrum; Thermo Fisher Scientific, Inc.). Experiments were repeated in triplicate.

Statistical analysis. All of the cell experiments were performed in triplicate with at least three independent biological replicates. GraphPad Prism (V5.0) software (GraphPad Software, Inc., La Jolla, CA, USA) was used for statistical analyses. Data are presented as the means \pm standard error of the mean from at least three independent values. Student's t-test or one-way analysis 
Table I. mRNA-specific primers used in reverse transcription-quantitative polymerase chain reaction.

\begin{tabular}{|c|c|c|}
\hline Gene & Sequence $\left(5^{\prime}-3^{\prime}\right)$ & $\mathrm{Ta}\left({ }^{\circ} \mathrm{C}\right.$ \\
\hline ms-SFMBT2 & $\begin{array}{l}\text { F: TAACTGCTGTCCTGCCTGCT } \\
\text { R: TGTGACATGGCCTACAGCTC }\end{array}$ & 61 \\
\hline ms-SOX9 & $\begin{array}{l}\text { F: TATGTGGATGTGTGCGTGTG } \\
\text { R: CCAGCCACAGCAGTGAGTAA }\end{array}$ & 61 \\
\hline ms-COL2A1 & $\begin{array}{l}\text { F: AGAGCGGAGACTACTGGATTG } \\
\text { R: CGTTAGCGGTGTTGGGAG }\end{array}$ & 61 \\
\hline ms-COL10A1 & $\begin{array}{l}\text { F: TGGGATGCCTCTTGTCAGTG } \\
\text { R: GTGGGCGTGCCATTCTTAT }\end{array}$ & 61 \\
\hline ms- $\beta$-actin & $\begin{array}{l}\text { F: AACAGTCCGCCTAGAAGCAC } \\
\text { R: CGTTGACATCCGTAAAGACC }\end{array}$ & 61 \\
\hline hsa-SFMBT2 & $\begin{array}{l}\text { F: ACGAAACAGGAGGAGGAGGAGAG } \\
\text { R: GGAAGGGTCAGAAGCAGGAGTG }\end{array}$ & 64 \\
\hline hsa-SOX9 & $\begin{array}{l}\text { F: CGCACATCAAGACGGAGCAG } \\
\text { R: TGTAGGTGAAGGTGGAGTAGAGG }\end{array}$ & 61 \\
\hline hsa-GAPDH & $\begin{array}{l}\text { F: CACCCACTCCTCCACCTTTG } \\
\text { R: CCACCACCCTGTTGCTGTAG }\end{array}$ & 61 \\
\hline
\end{tabular}

COL10A1, collagen type X $\alpha 1$ chain; COL2A1, collagen type II $\alpha 1$ chain; F, forward; hsa, Homo sapiens; ms, mouse; R, reverse; SFMBT2,Scm-like with four malignant brain tumor domains 2; SOX9, SRY-box 9; Ta, annealing temperature; F, forward; R, reverse.

of variance with Tukey's multiple comparison test were used to compare differences between the experimental groups. $\mathrm{P}<0.05$ was considered to indicate a statistically significant difference.

\section{Results}

SFMBT2 is abundantly expressed during the early stages of cartilage development. To examine the expression of SFMBT2 in articular cartilage, femoral head cartilages were surgically isolated from DA rats at D0, D21 and D42, which represent newborn/baby (0 years old), ablactation/child (5 years old) and juvenile (11 years old) developmental stages in humans, respectively (33). Immunohistochemical staining of the cartilage samples using a specific antibody against SFMBT2 revealed that the protein expression levels of SFMBT2 were markedly higher at D0 $(\sim 80 \%)$ and D21 $(\sim 63 \%)$, whereas the expression was significantly decreased at D42 ( 18\%) (P<0.05; Fig. 1).

SFMBT2 is upregulated during the proliferative phase of chondrogenesis. Chondrogenesis was induced in ATDC5 cells, and chondrocyte differentiation was verified through the expression of key chondrogenic markers, including SOX9, COL2A1 and COL10A1. The mRNA and protein expression levels were analyzed at specific time-points by RT-qPCR and western blotting, respectively. As shown in Fig. 2A, the mRNA expression levels of SFMBT2, SOX9 and COL2A1 were markedly increased on days 3 and 7 (proliferation), but were significantly decreased at day 14 (hypertrophy) compared with at day 7 by $>7$-, $>2$ - and $>16$-fold, respectively $(\mathrm{P}<0.05)$. COL10A 1 transcription remained low during the proliferative phase (days 3-7) but was increased at day 14 by $>3$-fold $(\mathrm{P}<0.05)$, compared with D0-D7. Western blotting results were
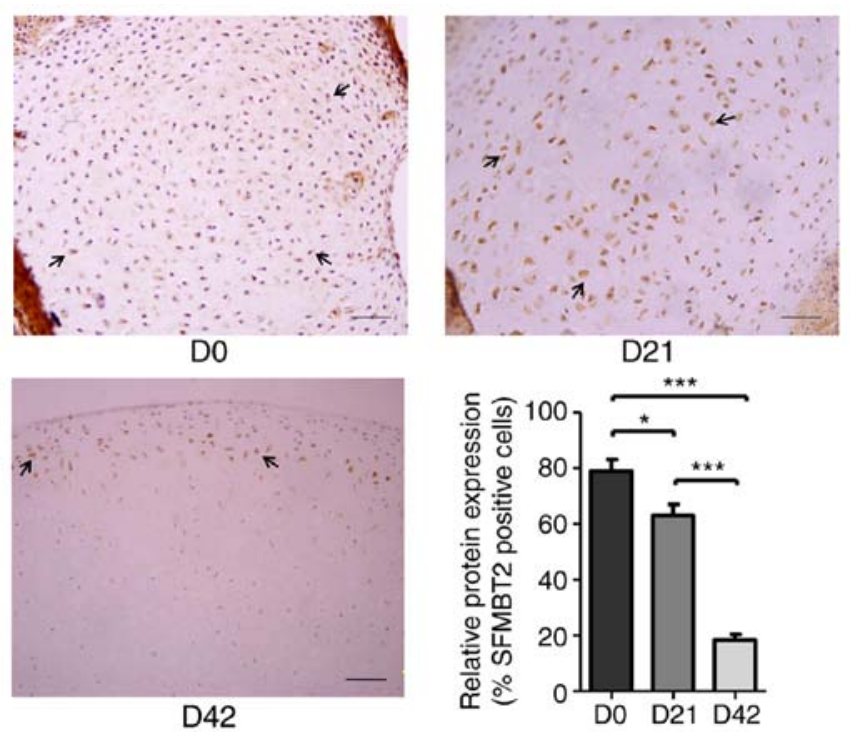

Figure 1. SFMBT2 expression in different stages of cartilage development. Representative immunohistochemical localization of SFMBT2 in the femoral head articular cartilages of dark agouti rats at D0 (upper left), D21 (upper right) and D42 (lower left). Arrows indicate SFMBT2-positive cells. Scale bar, $100 \mu \mathrm{m}$. The percentage of positive cells in the cartilage samples at the three developmental stages was analyzed (lower right). ${ }^{*} \mathrm{P}<0.05 ;{ }^{* * * *} \mathrm{P}<0.001$. D, day; SFMBT2, Scm-like with four malignant brain tumor domains 2 .

consistent with the RT-qPCR data (Fig. 2B). These findings indicated that SFMBT2 may be involved in the proliferation of chondrocytes during chondrogenesis.

SFMBT2 regulates SOX9 expression in human chondrocytes. The expression levels of SFMBT2 were suppressed in C28/I2 
Table II. Antibodies used in the present study.

\begin{tabular}{|c|c|c|c|c|c|}
\hline Antibody (origin) & Type & Species specificity & Cat. no. & Company & $\begin{array}{l}\text { Application } \\
\text { (dilution) }\end{array}$ \\
\hline SFMBT2 (Rb) & Polyclonal & Human, mouse & 25256-1-AP & $\begin{array}{l}\text { ProteinTech Group, Inc. } \\
\text { (Chicago, IL, USA) }\end{array}$ & WB $(1: 500)$ \\
\hline SFMBT2 (Rb) & Polyclonal & Human, rat & bs-21105R & BIOSS (Beijing, China) & IHC (1:50) \\
\hline SOX9 (Rb) & Monoclonal & Human, mouse & ab182579 & Abcam (Cambridge, MA, USA) & WB $(1: 1,000)$ \\
\hline COL2A1 (Rb) & Polyclonal & Human, mouse & BA0533 & $\begin{array}{l}\text { Wuhan Boster Biological } \\
\text { Technology, Ltd. (Wuhan, China) }\end{array}$ & WB $(1: 200)$ \\
\hline COL10A1 (Rb) & Polyclonal & Human, mouse & BA2023 & $\begin{array}{l}\text { Wuhan Boster Biological } \\
\text { Technology, Ltd }\end{array}$ & WB $(1: 500)$ \\
\hline GAPDH (Rb) & Polyclonal & Human, mouse & 10494-1-AP & ProteinTech Group, Inc. & WB $(1: 2,000)$ \\
\hline $\begin{array}{l}\text { HRP-conjugated IgG } \\
\text { (goat) }\end{array}$ & Polyclonal & Rabbit & 31460 & $\begin{array}{l}\text { Thermo Fisher Scientific, Inc. } \\
\text { (Waltham, MA, USA) }\end{array}$ & WB $(1: 2,000)$ \\
\hline
\end{tabular}

COL10A1, collagen type X $\alpha 1$ chain; COL2A1, collagen type II $\alpha 1$ chain; HRP, horseradish peroxidase; IgG, immunoglobulin G; IHC, immunohistochemistry; Rb, rabbit; SFMBT2, Scm-like with four malignant brain tumor domains 2; SOX9, SRY-box 9; WB, western blotting.

A

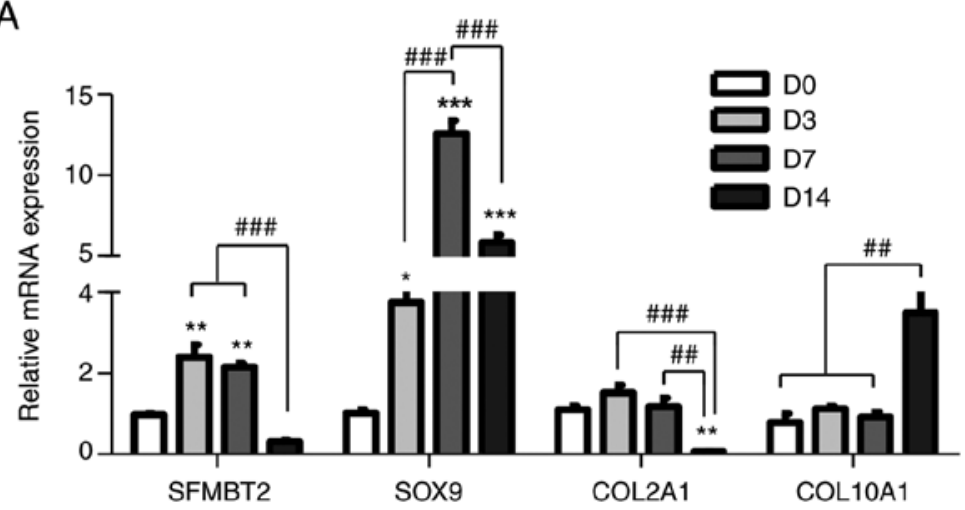

B
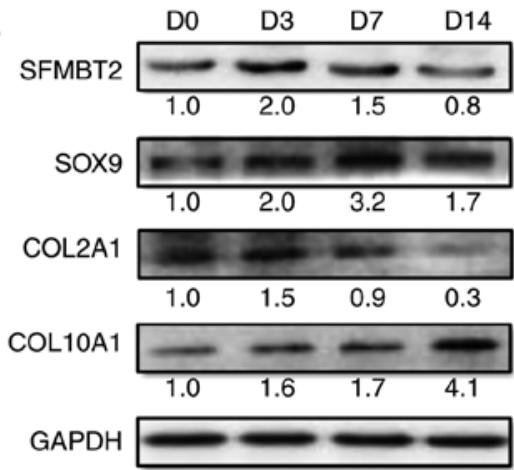

Figure 2. SFMBT2 expression during chondrogenesis. (A and B) Relative expression levels of SFMBT2 and chondrogenic markers (SOX9, COL2A1 and COL10A1) in ATDC5 cells cultured in insulin-transferrin-selenium-supplemented medium, as determined by (A) RT-qPCR and (B) western blotting. $\beta$-actin and GAPDH were used as internal controls in RT-qPCR and western blotting, respectively. ${ }^{*} \mathrm{P}<0.05,{ }^{* *} \mathrm{P}<0.01,{ }^{* * *} \mathrm{P}<0.001$ vs. the 'D0' group; ${ }^{\# \#} \mathrm{P}<0.01$,

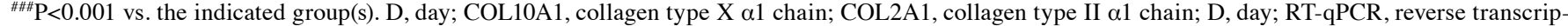
tion-quantitative polymerase chain reaction; SFMBT2, Scm-like with four malignant brain tumor domains 2; SOX9, SRY-box 9.

cells in response to si-SFMBT2. Endogenous SFMBT2 was knocked down $>70 \%$ by $80 \mathrm{nM}$ si-SFMBT2 (Fig. 3A and B). Decreased levels of SFMBT2 in C28/I2 cells led to a significant reduction in SOX9, both at the mRNA ( $>1.7$-fold; $\mathrm{P}<0.05)$ and protein levels $(>2$-fold; $\mathrm{P}<0.05)$, as determined by RT-qPCR and western blotting (Fig. 3A and B), respectively. Conversely, overexpression of SFMBT2 in C28/I2 cells was achieved by infecting the cells with ad-SFMBT2. Compared with ad-NC, chondrocytes infected with 400 MOI ad-SFMBT2 exhibited $>18$-fold higher levels of SFMBT2 $(\mathrm{P}<0.05)$, which resulted in significant upregulation of SOX9 at the mRNA (>2.4-fold; $\mathrm{P}<0.05)$ (Fig. 3C) and protein levels (>7-fold; $\mathrm{P}<0.05$ ) (Fig. 3D).

SFMBT2 suppresses SOX9 reduction in TNF- $\alpha$-treated C28/I2 cells. Different titers (MOIs) of ad-SFMBT2 were use to observe its effects on protein levels; as expected, ad-SFMBT2 upregulated SOX9 protein expression in a dose-dependent manner, as determined by western blotting (Fig. 4A). To investigate whether SOX9 could regulate SFMBT2, si-SOX9 was used. C28/I2 cells transfected with $60 \mathrm{nM}$ si-SOX9 exhibited a marked decrease $(>70 \%)$ in the protein expression levels of SOX9 compared with in cells transfected with si-NC. However, no change in the expression levels of SFMBT2 was observed (Fig. 4B), thus suggesting that SFMBT2 is located upstream of SOX9. Subsequently, SOX9 and SFMBT2 expression was examined in C28/I2 cells treated with IL-1 $\beta(10 \mathrm{ng} / \mathrm{ml})$ or TNF- $\alpha(10 \mathrm{ng} / \mathrm{ml})$. The results of a western blot analysis revealed that SOX9 expression was decreased in response to IL-1 $\beta$ and TNF- $\alpha$ treatment (by $>2$ and $>3$-fold, respectively). SFMBT2 expression was not significantly altered in response to IL-1 $\beta$; however, it was decreased by $>1.4$-fold in response to 24 h of treatment with TNF- $\alpha$ (Fig. 4C). Furthermore, C28/I2 cells were treated with $\mathrm{TNF}-\alpha$ for various durations. As shown 

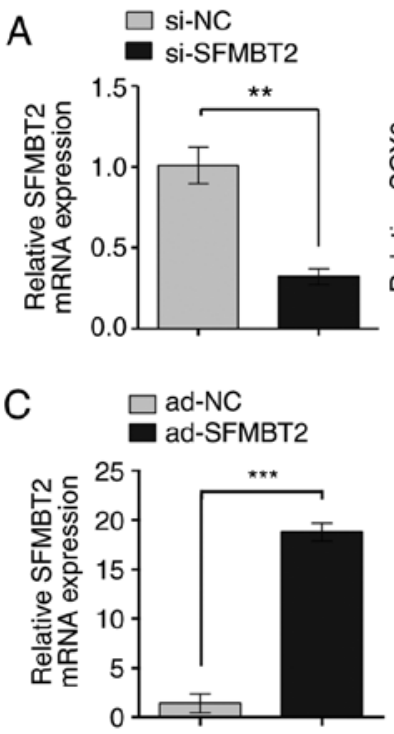
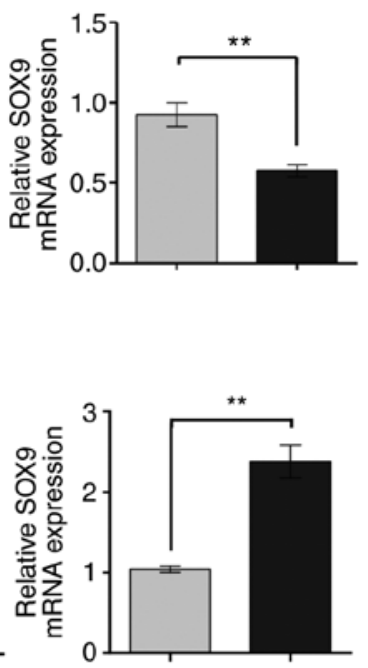

B

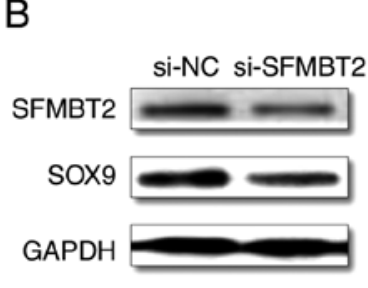

D

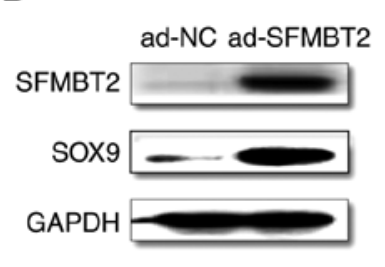

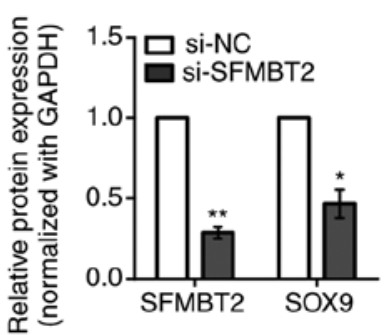

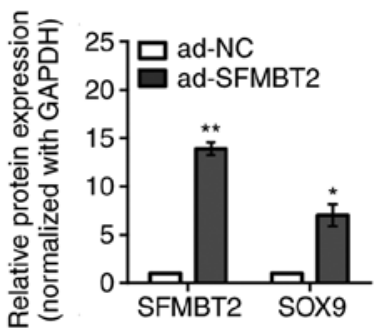

Figure 3. SFMBT2 positively regulates SOX9. Relative (A) mRNA and (B) protein expression levels of SFMBT2 and SOX9 in C28/I2 cells transfected with $80 \mathrm{nM}$ si-NC or si-SFMBT2, as measured by RT-qPCR and western blotting, respectively. Relative (C) mRNA and (D) protein expression levels of SFMBT2 and SOX9 in C28/I2 cells infected with ad-NC or ad-SFMBT2 (400 multiplicity of infection), as determined by RT-qPCR and western blotting, respectively. The C28/I2 cells were harvested for RNA and protein isolation at 24 and 48 h post-treatment with siRNA or adenoviral vector, respectively. GAPDH was used as the internal control for RT-qPCR and western blotting. ${ }^{*} \mathrm{P}<0.05 ;{ }^{* *} \mathrm{P}<0.01 ;{ }^{* * *} \mathrm{P}<0.001$. ad, adenovirus; NC, negative control; RT-qPCR, reverse transcription-quantitative polymerase chain reaction; SFMBT2, Scm-like with four malignant brain tumor domains 2 ; si, small interfering; SOX9, SRY-box 9.

in Fig. 4D, SOX9 expression was decreased in TNF- $\alpha$-treated chondrocytes in a time-dependent manner. However, infecting cells with 400 MOI ad-SFMBT2 prevented SOX9 reduction, and increased SOX9 protein levels in TNF- $\alpha$-treated chondrocytes.

SFMBT2 positively regulates chondrocyte proliferation. The proliferation of $\mathrm{C} 28 / \mathrm{I} 2$ chondrocytes was observed using the CCK-8. C28/12 cells were transfected/infected with si-SFMBT2 or ad-SFMBT2, alongside their respective controls. The results demonstrated that si-SFMBT2 transfection decreased the proliferation of chondrocytes compared with in the si-NC group (Fig. 5A). Conversely, chondrocytes infected with ad-SFMBT2 exhibited increased proliferation compared with in the ad-NC group, as measured at the indicated time intervals (Fig. 5B).

\section{Discussion}

A previous study reported that SFMBT2 is highly expressed in extra-embryonic tissues, such as the placenta and yolk sac, and is required for trophoblast maintenance and placental development (34). The present study analyzed the expression levels of SFMBT2 during cartilage development. For that purpose, DA rats were raised and sacrificed at the indicated time points. Immunohistochemical staining of femoral head cartilage specimens collected at various stages of cartilage development revealed that SFMBT2 was markedly expressed during the early stages of development (D0 and D21), thus suggesting that this gene may be involved in early cartilage development. Notably, SFMBT2 expression was reduced in hypertrophic chondrocytes, and at D42 its expression was confined to the proliferating zone, thus indicating that SFMBT2 may participate in chondrocyte proliferation. The downregulation of
SFMBT2 expression observed at D42 may indicate a lack of function of this gene in mature articular cartilage. However, due to the complex mechanism underlying maintenance of cartilage anabolic and catabolic homeostasis, certain levels of molecular factors are crucial to retain that balance. This was witnessed in our previous SFMBT2 knockdown experiments, where C28/I2 cells transfected with si-SFMBT2 displayed altered expression levels of metabolic marker genes, suggesting that downregulation of SFMBT2 may disturb the homeostatic balance of chondrocytes (30).

Chondrogenesis is a process by which cartilage is formed. It is primarily driven by the activity and expression of the transcription factor SOX9, which stimulates and maintains the expression of numerous cartilage-related genes, including COL2A1 and ACAN $(14,35)$. As a prerequisite for chondrogenesis in vivo, SOX9 activates COL2A1 transcription by binding to the first intron enhancer through its high mobility group DNA-binding domain $(36,37)$. Later phases of chondrogenesis are marked and regulated by the sequential activation of genes, including Indian hedgehog, runt-related transcription factor 2, COL10A1 and matrix metalloproteinase 13 (MMP13), and the simultaneous downregulation of SOX9 and COL2A1 (38). Following chondrogenesis, chondrocytes remain as resting cells forming the articular cartilage, or undergo proliferation, terminal differentiation to chondrocyte hypertrophy and apoptosis in a process termed endochondral ossification, whereby the hypertrophic cartilage is replaced by bone (39).

ATDC5 cells are derived from mouse teratocarcinoma cells (40) and are characterized as prechondrogenic cells that remain undifferentiated if cultured in regular medium. However, when grown in medium containing a chondrogenic differentiation supplement, these cells can replicate the events of chondrogenesis, from cell condensation to cartilage nodule 
A

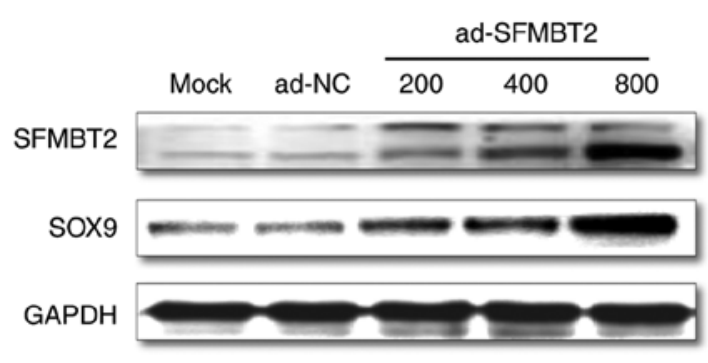

B

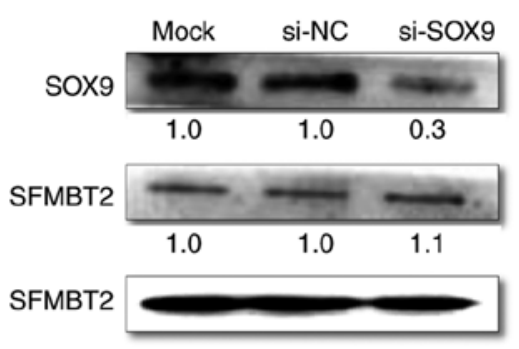

D

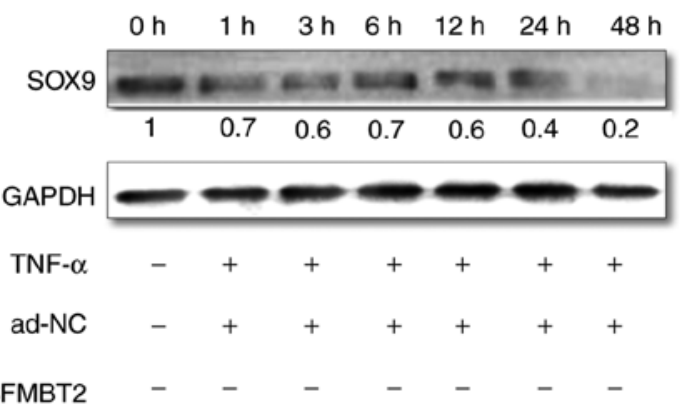

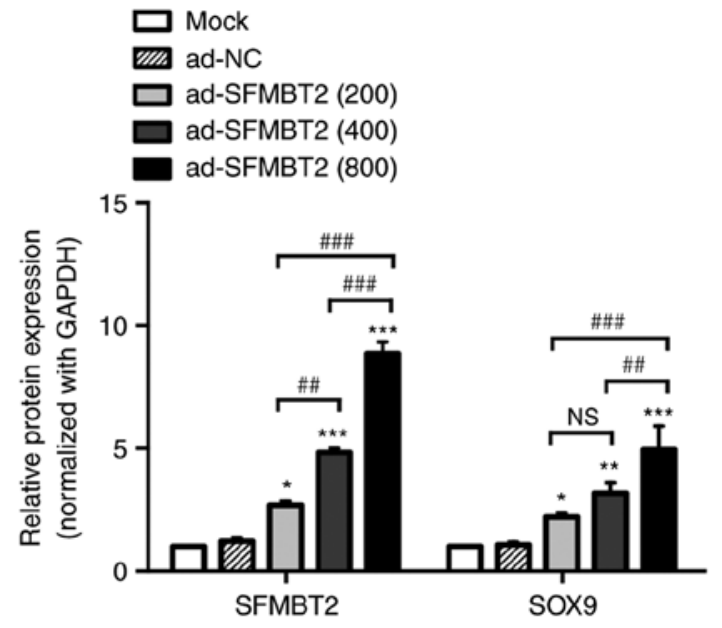

$\mathrm{C}$
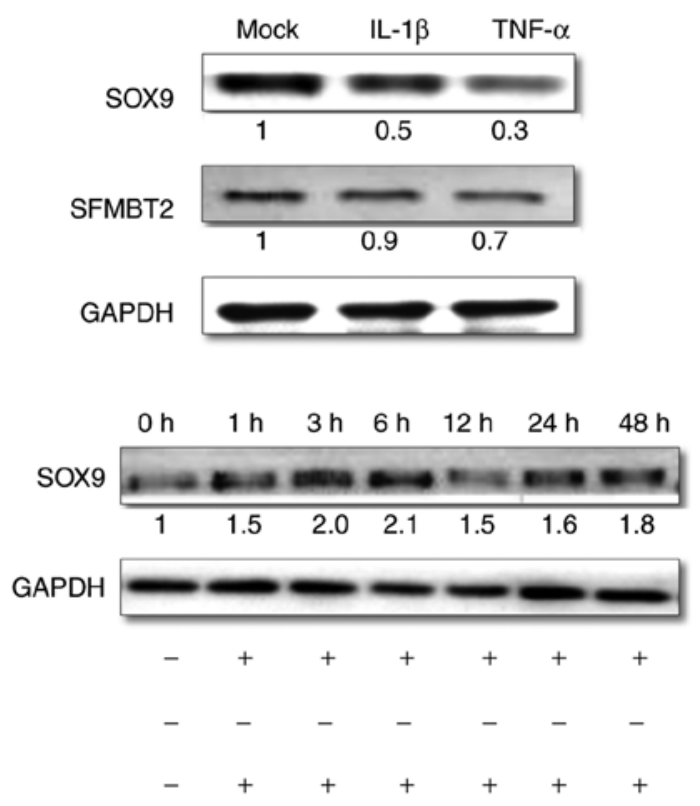

Figure 4. Exogenous SFMBT2 attenuates the reduction in SOX9 expression in C28/I2 chondrocytes under TNF- $\alpha$ treatment. (A) Left panel, dose-dependent effect of SFMBT2 overexpression on SOX9 in C28/12 cells harvested at 48 h post-infection, as determined by western blotting (ad-SFMBT2 was used at 200, 400 and 800 MOI, whereas ad-NC was used at $400 \mathrm{MOI}$ ). Right panel, Statistical analysis of the relative protein expression levels normalized to GAPDH. (B) Relative protein expression levels of SOX9 and SFMBT2 in C28/I2 cells transfected with $60 \mathrm{nM}$ si-NC or si-SOX9 and harvested $48 \mathrm{~h}$ post-transfection, as analyzed by western blotting. (C) Effects of IL-1 $\beta$ and TNF- $\alpha$ on the expression levels of SOX 9 and SFMBT2 in C28/I 2 cells harvested 48 h post-treatment. (D) Relative protein expression levels of SOX9 in C28/I2 cells infected with ad-NC or ad-SFMBT2 (400 MOI) for $24 \mathrm{~h}$ and incubated with TNF- $\alpha$ (10 ng/ml). The cells were harvested for protein isolation at the indicated time points post-TNF- $\alpha$ treatment and were analyzed by western blotting. GAPDH was used as the internal control in western blot analyses. ${ }^{*} \mathrm{P}<0.05,{ }^{* *} \mathrm{P}<0.01,{ }^{* * *} \mathrm{P}<0.001$ vs. the Mock and ad-NC groups; ${ }^{\# \#} \mathrm{P}<0.01,{ }^{\# \# \#} \mathrm{P}<0.001$ vs. the indicated group. NS, non-significant. ad, adenovirus; IL, interleukin; MOI, multiplicity of infection; NC, negative control; RT-qPCR, reverse transcription-quantitative polymerase chain reaction; SFMBT2, Scm-like with four malignant brain tumor domains 2; si, small interfering; SOX9, SRY-box 9; TNF, tumor necrosis factor.

formation $(41,42)$. Therefore, the ATDC5 cell line is considered a promising in vitro cell model to study the sequential events of cartilage formation and the factors that influence chondrocyte behaviors during chondrogenesis. In the present study, the expression levels of SFMBT2 were increased in chondrogenic ATDC5 cells during the early phase of chondrogenesis (proliferating chondrocytes) alongside SOX9 and COL2A1, but were decreased in the later phases (hypertrophic differentiation) when COL10A1 was upregulated, indicating that SFMBT2 may be involved in the proliferation of chondrocytes. The high levels of COL10A1 at D14 are in accordance with hypertrophic degeneration, which physiologically precedes endochondral ossification. Under normal physiological conditions, mature articular cartilage behaves quite differently; SOX9 and COL2A1 levels are maintained even in the presence of low cellular turnover. In general, in normal tissues, cellular proliferation and differentiation are inversely related. The chondrocytes in adult human cartilage are normally quiescent and maintain the matrix in a low turnover state. Healthy hyaline cartilage does not express high levels of COL10A1 and has a very low rate of proliferation, simultaneously maintaining high SOX9 and COL2A1 levels. Conversely, during OA development, modifications towards hypertrophy take place (43-47).

SFMBT2 expression during the process of chondrogenesis was almost consistent with that of SOX9. Therefore, it was 

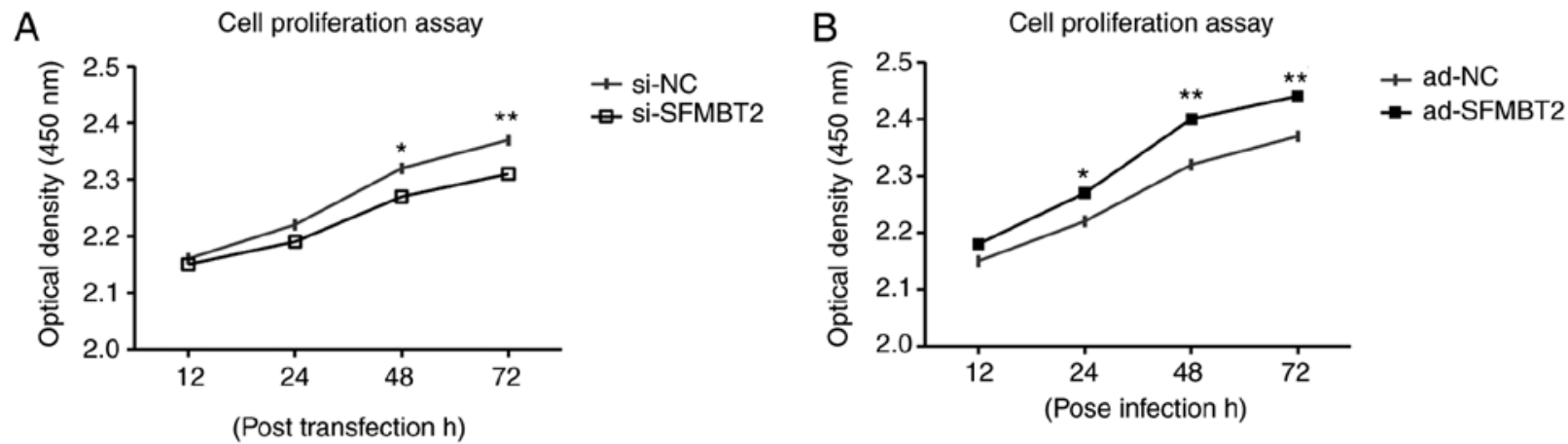

Figure 5. SFMBT2 positively regulates chondrocyte proliferation. (A) Proliferation of C28/I2 cells transfected with si-NC or si-SFMBT2, as determined 12 , 24, 48 and $72 \mathrm{~h}$ post-transfection by CCK-8 assay. (B) Chondrocytes were infected with ad-NC or ad-SFMBT2 for various durations (12, 24, 48 and $72 \mathrm{~h}$ ), and cell proliferation was determined by CCK-8 assay. ${ }^{*} \mathrm{P}<0.05 ;{ }^{* *} \mathrm{P}<0.01$. ad, adenovirus; CCK, cell counting kit; NC, negative control; SFMBT2, Scm-like with four malignant brain tumor domains 2 ; si, small interfering.

hypothesized that SFMBT2 may affect SOX9 expression in articular chondrocyte. The present study examined the effects of SFMBT2 knockdown and overexpression in C28/I2 cells using siRNA and adenoviral vectors, respectively. In mature articular chondrocytes, following SFMBT2 knockdown in vitro, SOX9 was significantly downregulated at the mRNA and protein levels, whereas overexpression of SFMBT2 upregulated SOX9. SOX9 is a pivotal transcriptional regulator, which is essential for chondrocyte phenotypic stability, differentiation (13) and proliferation (36). Downregulation of SOX9 may induce angiogenesis, cartilage resorption, and formation of bone marrow and endochondral bone trabeculae (22), which are associated with the progression of OA. The present study also investigated whether SOX9 could regulate SFMBT2 expression. For that purpose, SOX9 expression in C28/I2 cells was suppressed by targeting SOX9 mRNA using a specific siRNA sequence; however, si-SOX9 did not affect SFMBT2 expression levels in chondrocytes, indicating that SFMBT2 is upstream of SOX9.

Studies on chondrocyte behavior in OA suggest that at different stages and/or locations within articular cartilage, the processes of cartilage matrix anabolism and catabolism are regulated through coordinated mechanisms that are not fully understood. The degradation of articular cartilage due to catabolic behavior of chondrocytes, thus leading to the destruction of ECM components, is a characteristic of OA $(48,49)$. In OA, the normally quiescent chondrocytes undergo a phenotypic shift and become 'activated' cells, characterized by cell proliferation, cluster formation, and increased production of matrix proteins and matrix-degrading enzymes (49). It is generally considered that inflammation is absent or weakly present in OA (50); however, numerous studies have confirmed the presence of immune cells and proinflammatory cytokines within the synovial tissues of patients with OA (51-54). The most acknowledged background theory is that the cartilage fragments and the degraded products fall into the joint and come into contact with the synovium (50). Considering them foreign bodies, synovial cells produce inflammatory cytokines in response to these cartilage fragments, which results in further activation of chondrocytes, the production of matrix-degrading enzymes [i.e. MMPs and a disintegrin and metalloproteinase with thrombospondin motifs (ADAMTSs)] and subsequent increased cartilage destruction (55).
Numerous studies have confirmed the upregulation of IL-1 $\beta$ and TNF- $\alpha$ in OA joints (51-54). Nuclear factor (NF)- $\kappa B$, which is a negative regulator of SOX9 expression, is mainly regulated by TNF- $\alpha$ and IL-1 $\beta$ (56). Activation of NF- $\mathrm{kB}$ and other inflammatory pathways, such as the mitogen-activated protein kinase signaling pathway, is required for chondrocytes to express MMPs, ADAMTSs and inflammatory cytokines $(44,57)$. In vitro studies have suggested that NF- $\kappa B$ is an upstream inducer of hypoxia-inducible factor- $2 \alpha$, which is another transactivator of genes expressed in hypertrophic chondrocytes, including COL10A1, MMP13 and vascular endothelial growth factor (58). These findings suggest that the anabolic-catabolic balance is under the influence of a complex network of signals that regulate chondrocyte behavior and tissue homeostasis (59). Catabolic activity is higher in OA (60), and due to their low turnover rate, chondrocytes are not able to substitute the degraded ECM (61). However, increased production of collagen type II and ACAN is commonly considered an early response to cartilage degeneration in in vivo and in vitro OA models $(62,63)$. SOX9 is a potent transcriptional activator of COL2A1, which participates in cartilage development and can maintain the chondrocyte phenotype $(13,20,64,65)$. When C28/I2 cells were incubated with various concentrations (MOIs) of ad-SFMBT2, SOX9 protein levels were elevated in a dose-dependent manner compared with in the ad-NC group. Furthermore, TNF- $\alpha$ treatment reduced SOX9 protein levels in C28/I2 cells. Infection with ad-SFMBT2 not only protected the cells from a reduction in SOX9 levels, but also increased its expression in a time-dependent manner.

The present study investigated whether alterations in SFMBT2 levels could affect the proliferation of C28/I2 cells. SFMBT2 was knocked down in chondrocytes, which were subjected to a cell proliferation assay using the CCK- 8 . SFMBT2 knockdown decreased cell proliferation compared with in the si-NC-transfected cells. Conversely, the ad-SFMBT2 group displayed an increased proliferative rate compared with in the ad-NC group. These results suggested that SFMBT2 may function as a positive regulator of cell proliferation in human chondrocytes.

In conclusion, the present study revealed that SFMBT2 could target and regulate the expression of SOX9, and could protect chondrocytes from losing SOX9 expression under 
TNF- $\alpha$ treatment. SFMBT2 also promoted chondrocyte proliferation. Further studies may reveal this promising molecule as a potential therapeutic agent against OA.

\section{Acknowledgements}

The C28/I2 cell line was provided by Prof. Junling Cao (Institute of Endemic Diseases, Xi'an Jiaotong University, Health Science Center).

\section{Funding}

The present study was supported by grants from the National Natural Science Foundation of China (grant nos. 81371986, 81772410 and 81301598 ), the Shaanxi Province Natural Science Foundation (grant no. S2016YFJM1171) and the Fundamental Research Funds for the Central Universities (grant no. xjj2015073).

\section{Availability of data and materials}

The datasets used and/or analyzed during this study are available from the corresponding author on reasonable request.

\section{Authors' contributions}

SH designed and executed the experiments, analyzed the data and wrote the manuscript. MS and YG helped in the analyses and interpretation of data. NM, YZ, YY, NH, EO, AS, MS, FZ and $\mathrm{YH}$ helped perform the experiments. JS and SL conceived and designed the study, critically reviewed and drafted the manuscript and take responsibility for the integrity of the work as a whole. All authors read and approved the final manuscript.

\section{Ethics approval and consent to participate}

The animal study was approved by the Institutional Animal Ethics Committee of Xi'an Jiaotong University, Health Science Center.

\section{Patient consent for publication}

Not applicable.

\section{Competing interests}

The authors declare that they have no competing interests.

\section{References}

1. Sandell LJ, Sugai JV and Trippel SB: Expression of collagens I, II, X, and XI and aggrecan mRNAs by bovine growth plate chondrocytes in situ. J Orthop Res 12: 1-14, 1994.

2. Cleland KA, James MJ, Neumann MA, Gibson RA and Cleland LG: Differences in fatty acid composition of immature and mature articular cartilage in humans and sheep. Lipids 30: 949-953, 1995.

3. Muir H: The chondrocyte, architect of cartilage. Biomechanics, structure, function and molecular biology of cartilage matrix macromolecules. Bioessays 17: 1039-1048, 1995.

4. Kinner B, Capito RM and Spector M: Regeneration of articular cartilage. Adv Biochem Eng Biotechnol 94: 91-123, 2005.

5. Goldring MB, Tsuchimochi $\mathrm{K}$ and Ijiri K: The control of chondrogenesis. J Cell Biochem 97: 33-44, 2006.
6. Aigner T, Zhu Y, Chansky HH, Matsen FA III, Maloney WJ and Sandell LJ: Reexpression of type IIA procollagen by adult articular chondrocytes in osteoarthritic cartilage. Arthritis Rheum 42: 1443-1450, 1999.

7. Cheng A and Genever PG: SOX9 determines RUNX2 transactivity by directing intracellular degradation. J Bone Miner Res 25: 2680-2689, 2010.

8. Yamashita S, Andoh M, Ueno-Kudoh H, Sato T, Miyaki S and Asahara H: Sox9 directly promotes Bapx1 gene expression to repress Runx2 in chondrocytes. Exp Cell Res 315: 2231-2240, 2009.

9. Chen H, Ghori-Javed FY, Rashid H, Adhami MD, Serra R, Gutierrez SE and Javed A: Runx2 regulates endochondral ossification through control of chondrocyte proliferation and differentiation. J Bone Miner Res 29: 2653-2665, 2014.

10. Ulrich C, Rolauffs B, Abele H, Bonin M, Nieselt K, Hart ML and Aicher WK: Low osteogenic differentiation potential of placenta-derived mesenchymal stromal cells correlates with low expression of the transcription factors Runx2 and Twist2. Stem Cells Dev 22: 2859-2872, 2013.

11. Buckwalter JA and Mankin HJ: Articular cartilage: Tissue design and chondrocyte-matrix interactions. Instr Course Lect 47: 477-486, 1998.

12. Goldring MB and Goldring SR: Osteoarthritis. J Cell Physiol 213: 626-634, 2007.

13. Bi W, Deng JM, Zhang Z, Behringer RR and de Crombrugghe B: Sox9 is required for cartilage formation. Nat Genet 22: 85-89, 1999.

14. Akiyama H, Chaboissier MC, Martin JF, Schedl A and de Crombrugghe B: The transcription factor Sox 9 has essential roles in successive steps of the chondrocyte differentiation pathway and is required for expression of Sox 5 and Sox6. Genes Dev 16: 2813-2828, 2002.

15. Furumatsu T, Tsuda M, Taniguchi N, Tajima Y and Asahara H: Smad3 induces chondrogenesis through the activation of SOX 9 via CREB-binding protein/p300 recruitment. J Biol Chem 280: 8343-8350, 2005.

16. Akiyama $\mathrm{H}$ and Lefebvre $\mathrm{V}$ : Unraveling the transcriptional regulatory machinery in chondrogenesis. J Bone Miner Metab 29: 390-395, 2011.

17. Linn FC and Sokoloff L: Movement and Composition of interstitial fluid of cartilage. Arthritis Rheum 8: 481-494, 1965.

18. Yamashita S, Miyaki S, Kato Y, Yokoyama S, Sato T, Barrionuevo F, Akiyama H, Scherer G, Takada S and Asahara H: L-Sox 5 and Sox6 proteins enhance chondrogenic miR-140 microRNA expression by strengthening dimeric Sox 9 activity. J Biol Chem 287: 22206-22215, 2012.

19. Lefebvre V, Behringer RR and de Crombrugghe B: L-Sox5, Sox6 and Sox 9 control essential steps of the chondrocyte differentiation pathway. Osteoarthritis Cartilage 9 (Suppl A): S69-S75, 2001.

20. Ng LJ, Wheatley S, Muscat GE, Conway-Campbell J, Bowles J, Wright E, Bell DM, Tam PP, Cheah KS and Koopman P: SOX9 binds DNA, activates transcription, and coexpresses with type II collagen during chondrogenesis in the mouse. Dev Biol 183: 108-121, 1997.

21. Zhao Q, Eberspaecher H, Lefebvre V and De Crombrugghe B: Parallel expression of Sox 9 and Col2a1 in cells undergoing chondrogenesis. Dev Dyn 209: 377-386, 1997.

22. Hattori T, Muller C, Gebhard S, Bauer E, Pausch F, Schlund B, Bösl MR, Hess A, Surmann-Schmitt C, von der Mark H, et al: SOX9 is a major negative regulator of cartilage vascularization, bone marrow formation and endochondral ossification. Development 137: 901-911, 2010.

23. Foster JW, Dominguez-Steglich MA, Guioli S, Kwok C, Weller PA, Stevanović M, Weissenbach J, Mansour S, Young ID, Goodfellow PN, et al: Campomelic dysplasia and autosomal sex reversal caused by mutations in an SRY-related gene. Nature 372: 525-530, 1994.

24. Bracken AP and Helin K: Polycomb group proteins: Navigators of lineage pathways led astray in cancer. Nat Rev Cancer 9: 773-784, 2009.

25. Tsai HC and Baylin SB: Cancer epigenetics: Linking basic biology to clinical medicine. Cell Res 21: 502-517, 2011.

26. Klymenko T, Papp B, Fischle W, Köcher T, Schelder M, Fritsch C, Wild B, Wilm M and Müller J: A Polycomb group protein complex with sequence-specific DNA-binding and selective methyl-lysine-binding activities. Genes Dev 20: 1110-1122, 2006.

27. Lee K, Na W, Maeng JH, Wu H and Ju BG: Regulation of DU145 prostate cancer cell growth by Scm-like with four mbt domains 2 . J Biosci 38: 105-112, 2013. 
28. Wu S, Trievel RC and Rice JC: Human SFMBT is a transcriptional repressor protein that selectively binds the N-terminal tail of histone H3. FEBS Lett 581: 3289-3296, 2007.

29. Wang W, Zhong B, Sun J, Cao J, Tian J, Zhong N, Zhao W, Tian L, $\mathrm{Xu}$ P, Guo D, et al: Down-regulated HS6ST2 in osteoarthritis and Kashin-Beck disease inhibits cell viability and influences expression of the genes relevant to aggrecan metabolism of human chondrocytes. Rheumatology (Oxford) 50: 2176-2186, 2011.

30. Hussain S, Sun M, Min Z, Guo Y, Xu J, Mushtaq N, Heng L, Huang $\mathrm{H}$, Zhao Y, Yuan Y, et al: Down-regulated in OA cartilage, SFMBT2 contributes to NF- $\kappa \mathrm{B}$ mediated ECM degradation. J Cell Mol Med: Aug 22, 2018 (Epub ahead of print). doi: $10.1111 / \mathrm{jcmm} .13826$.

31. Livak KJ and Schmittgen TD: Analysis of relative gene expression data using real-time quantitative PCR and the 2(-Delta Delta C(T)) method. Methods 25: 402-408, 2001.

32. Wei X, Peng G, Zheng S and Wu X: Differentiation of umbilical cord mesenchymal stem cells into steroidogenic cells in comparison to bone marrow mesenchymal stem cells. Cell Prolif 45: 101-110, 2012

33. Fox JG, Cohen BJ and Loew FM: Laboratory Animal Medicine. Academic Press, Inc., Harcourt Brace Jovanovich, San Diego, CA, 1984.

34. Miri K, Latham K, Panning B, Zhong Z, Andersen A and Varmuza S: The imprinted polycomb group gene Sfmbt 2 is required for trophoblast maintenance and placenta development. Development 140: 4480-4489, 2013.

35. Horton WA: Skeletal development: Insights from targeting the mouse genome. Lancet 362: 560-569, 2003.

36. Lefebvre $\mathrm{V}, \mathrm{Li} \mathrm{P}$ and de Crombrugghe $\mathrm{B}$ : A new long form of Sox 5 (L-Sox5), Sox6 and Sox9 are coexpressed in chondrogenesis and cooperatively activate the type II collagen gene. Embo J 17: 5718-5733, 1998.

37. Leung KK, Ng LJ, Ho KK, Tam PP and Cheah KS: Different cis-regulatory DNA elements mediate developmental stage- and tissue-specific expression of the human COL2A1 gene in transgenic mice. J Cell Biol 141: 1291-1300, 1998.

38. Zuscik MJ, Hilton MJ, Zhang X, Chen D and O'Keefe RJ: Regulation of chondrogenesis and chondrocyte differentiation by stress. J Clin Invest 118: 429-438, 2008

39. Lefebvre V and Smits P: Transcriptional control of chondrocyte fate and differentiation. Birth Defects Res C Embryo Today 75: 200-212, 2005.

40. Atsumi T, Miwa Y, Kimata K and Ikawa Y: A chondrogenic cell line derived from a differentiating culture of AT805 teratocarcinoma cells. Cell Differ Dev 30: 109-116, 1990.

41. Shukunami C, Shigeno C, Atsumi T, Ishizeki K, Suzuki F and Hiraki Y: Chondrogenic differentiation of clonal mouse embryonic cell line ATDC5 in vitro: Differentiation-dependent gene expression of parathyroid hormone $(\mathrm{PTH}) / \mathrm{PTH}$-related peptide receptor. J Cell Biol 133: 457-468, 1996.

42. Shukunami C, Ohta Y, Sakuda M and Hiraki Y: Sequential progression of the differentiation program by bone morphogenetic protein-2 in chondrogenic cell line ATDC5. Exp Cell Res 241: 1-11, 1998.

43. Henry SP, Liang S, Akdemir KC and de Crombrugghe B: The postnatal role of Sox 9 in cartilage. J Bone Miner Res 27: 2511-2525, 2012.

44. Goldring MB: Chondrogenesis, chondrocyte differentiation, and articular cartilage metabolism in health and osteoarthritis. Ther Adv Musculoskelet Dis 4: 269-285, 2012.

45. Salminen H, Vuorio E and Saamanen AM: Expression of Sox9 and type IIA procollagen during attempted repair of articular cartilage damage in a transgenic mouse model of osteoarthritis. Arthritis Rheum 44: 947-955, 2001

46. Binette F, McQuaid DP, Haudenschild DR, Yaeger PC, McPherson JM and Tubo R: Expression of a stable articular cartilage phenotype without evidence of hypertrophy by adult human articular chondrocytes in vitro. J Orthop Res 16: 207-216, 1998.
47. Hoyland JA, Thomas JT, Donn R, Marriott A, Ayad S, Boot-Handford RP, Grant ME and Freemont AJ: Distribution of type X collagen mRNA in normal and osteoarthritic human cartilage. Bone Miner 15: 151-163, 1991

48. Goldring MB, Otero M, Tsuchimochi K, Ijiri K and Li Y. Defining the roles of inflammatory and anabolic cytokines in cartilage metabolism. Ann Rheum Dis 67 (Suppl 3): iii75-iii82, 2008.

49. Goldring MB and Marcu KB: Cartilage homeostasis in health and rheumatic diseases. Arthritis Res Ther 11: 224, 2009.

50. Berenbaum F: Osteoarthritis as an inflammatory disease (osteoarthritis is not osteoarthrosis!). Osteoarthritis Cartilage 21: 16-21, 2013

51. Pelletier JP and Martel-Pelletier J: Evidence for the involvement of interleukin 1 in human osteoarthritic cartilage degradation: Protective effect of NSAID. J Rheumatol 18: 19-27, 1989.

52. Farahat MN, Yanni G, Poston R and Panayi GS: Cytokine expression in synovial membranes of patients with rheumatoid arthritis and osteoarthritis. Ann Rheum Dis 52: 870-875, 1993.

53. Tetlow LC, Adlam DJ and Woolley DE: Matrix metalloproteinase and proinflammatory cytokine production by chondrocytes of human osteoarthritic cartilage: Associations with degenerative changes. Arthritis Rheum 44: 585-594, 2001.

54. Muñoz-Valle JF, Oregón-Romero E, Rangel-Villalobos H, Martínez-Bonilla GE, Castañeda-Saucedo E, Salgado-Goytia L, Leyva-Vázquez MA, Illades-Aguiar B, Alarcón-Romero Ldel C, Espinoza-Rojo $\mathrm{M}$ and Parra-Rojas I: High expression of TNF alpha is associated with -308 and -238 TNF alpha polymorphisms in knee osteoarthritis. Clin Exp Med 14: 61-67, 2014.

55. Kim KI, Park YS and Im GI: Changes in the epigenetic status of the SOX-9 promoter in human osteoarthritic cartilage. J Bone Miner Res 28: 1050-1060, 2013.

56. Sitcheran R, Cogswell PC and Baldwin AS Jr: NF-kappaB mediates inhibition of mesenchymal cell differentiation through a posttranscriptional gene silencing mechanism. Genes Dev 17: 2368-2373, 2003.

57. Schroeppel JP, Crist JD, Anderson HC and Wang J: Molecular regulation of articular chondrocyte function and its significance in osteoarthritis. Histol Histopathol 26: 377-394, 2011.

58. Yang S, Kim J, Ryu JH, Oh H, Chun CH, Kim BJ, Min BH and Chun JS: Hypoxia-inducible factor-2alpha is a catabolic regulator of osteoarthritic cartilage destruction. Nat Med 16: 687-693, 2010.

59. Mueller MB and Tuan RS: Anabolic/Catabolic balance in pathogenesis of osteoarthritis: Identifying molecular targets. PM R 3 (Suppl 1): S3-S11, 2011.

60. Madry H, Luyten FP and Facchini A: Biological aspects of early osteoarthritis. Knee Surg Sports Traumatol Arthrosc 20: 407-422, 2012

61. Wang M, Shen J, Jin H, Im HJ, Sandy J and Chen D: Recent progress in understanding molecular mechanisms of cartilage degeneration during osteoarthritis. Ann N Y Acad Sci 1240: 61-69, 2011

62. Matyas JR, Adams ME, Huang D and Sandell LJ: Discoordinate gene expression of aggrecan and type II collagen in experimental osteoarthritis. Arthritis Rheum 38: 420-425, 1995.

63. Cs-Szabó G, Melching LI, Roughley PJ and Glant TT: Changes in messenger RNA and protein levels of proteoglycans and link protein in human osteoarthritic cartilage samples. Arthritis Rheum 40: 1037-1045, 1997.

64. Lefebvre $\mathrm{V}$ and de Crombrugghe $\mathrm{B}$ : Toward understanding SOX9 function in chondrocyte differentiation. Matrix Biol 16: $529-540,1998$

65. Lefebvre V, Huang W, Harley VR, Goodfellow PN and de Crombrugghe B: SOX9 is a potent activator of the chondrocyte-specific enhancer of the pro alpha1(II) collagen gene. Mol Cell Biol 17: 2336-2346, 1997. 